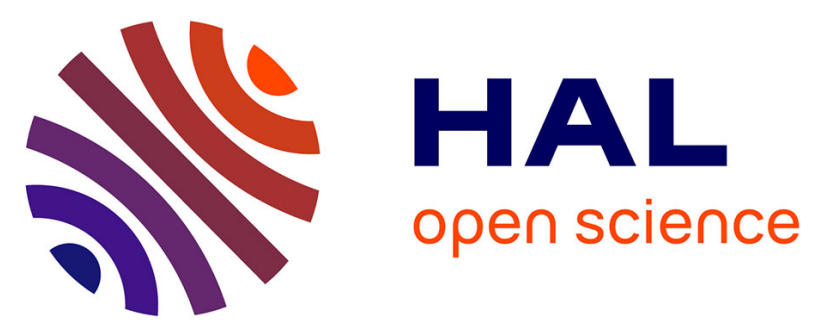

\title{
Three-Dimensional Silver Nanoparticle Formation Using Femtosecond Laser Irradiation in Phosphate Glasses: Analogy with Photography
}

\author{
Nicolas Marquestaut, Yannick Petit, Arnaud Royon, Patrick Mounaix, \\ Thierry Cardinal, Lionel Canioni
}

\section{To cite this version:}

Nicolas Marquestaut, Yannick Petit, Arnaud Royon, Patrick Mounaix, Thierry Cardinal, et al.. Three-Dimensional Silver Nanoparticle Formation Using Femtosecond Laser Irradiation in Phosphate Glasses: Analogy with Photography. Advanced Functional Materials, 2014, 24 (37), pp.5824-5832. 10.1002/adfm.201401103 . hal-01052820

\section{HAL Id: hal-01052820 https://hal.science/hal-01052820}

Submitted on 23 Feb 2016

HAL is a multi-disciplinary open access archive for the deposit and dissemination of scientific research documents, whether they are published or not. The documents may come from teaching and research institutions in France or abroad, or from public or private research centers.
L'archive ouverte pluridisciplinaire HAL, est destinée au dépôt et à la diffusion de documents scientifiques de niveau recherche, publiés ou non, émanant des établissements d'enseignement et de recherche français ou étrangers, des laboratoires publics ou privés.

\section{(ㅇ)(1) $\$$}

Distributed under a Creative Commons Attribution - NonCommerciall 4.0 International 


\title{
Three-Dimensional Silver Nanoparticle Formation Using Femtosecond Laser Irradiation in Phosphate Glasses: Analogy with Photography
}

\author{
N. Marquestaut, * Y. Petit, A. Royon, P. Mounaix, T. Cardinal, and L. Canioni
}

\begin{abstract}
Three-dimensional patterning of metal-dielectric composites is achieved at the sub-micrometer scale in glass. A high silver-content zinc-phosphate optical transparent glass is structured by means of a near-infrared femtosecond laser via non-linear absorption processes. The silver ions embedded in glass photochemically react under controlled laser irradiation, inducing ion clustering and the formation of nucleation centers. A subsequent thermal development causes silver reduction and growth into metallic nanoparticles. A very strong surface plasmon resonance is measured, revealing the very high concentration of metallic particles. The direct laser writing process enables inscriptions of silver nanoparticle domains well below the diffraction limit $(\sim 100 \mathrm{~nm})$. A very detailed parametric study is given, leading to the first thorough understanding of the ultrafast laser-induced photochemistry in silver-containing glass materials. These nanostructures are considered promising candidates for the design of highly efficient sensing devices via plasmonics and, beyond that, for photonics and metamaterials applications.
\end{abstract}

controlled depths, is permitted thanks to non-linear absorption processes.

Within this context, pulsed lasers in combination with tailored dielectric materials containing photosensitive agents can initiate local photochemical processes and the formation of metallic nanoparticles (NPs). ${ }^{[3,4]}$ The optical properties of the resulting metal-dielectric nanocomposites are determined, among other properties, by the surface plasmon resonance (SPR) of the metallic NPs. Depending on the targeted application, these SPRs can be tuned within a broad spectral range from visible to near-infrared (NIR). ${ }^{[5]}$ Furthermore, the SPR band is also strongly dependent on the size distribution, the shape, and the concentration of the NPs as well as the dielectric matrix. Thus, laserbased techniques in matrices such as glass permit the modification of these NP fea-

\section{Introduction}

Optical properties of materials can be tailored by using ultrafast and highly intense laser sources, opening routes towards the design of nanocomposite devices, thanks to various phenomena including oxido-reduction, diffusion, aggregation or phase transition processes. ${ }^{[1]}$ Among several lithographic techniques, femtosecond (fs) direct laser writing (DLW), considered a maskless patterning process, presents numerous advantages over the usual techniques, such as electron-beam lithography. DLW offers rapid patterning at sub-micrometer resolutions, with flexibility, ease of use, and cost effectiveness. ${ }^{[2]}$ At last, true 3D structuring inside optical transparent materials, at

Dr. N. Marquestaut, Dr. A. Royon,

Dr. P. Mounaix, Prof. L. Canioni

Laboratoire Ondes et Matière d'Aquitaine

University of Bordeaux

351 Cours de la Libération

33405, Talence, France

E-mail: nicolas.marquestaut@u-bordeaux.fr

Dr. Y. Petit, Dr. T. Cardinal

Institut de Chimie de la Matière Condensée de Bordeaux

University of Bordeaux

87 Avenue du Docteur Schweitzer

33608, Pessac, France tures and provide a very powerful and flexible tool to control and optimize the linear and non-linear optical properties of metal-dielectric nanocomposites. Practical advances in the past recent years in the metamaterials and plasmonics fields have also pointed out the interest in patterning metals or shaping particles in three dimensions inside transparent media such as glasses ${ }^{[6]}$ or polymers ${ }^{[7]}$ when accurate control of the spatial shaping and/or orientation of the NPs can be achieved.

Phosphate glasses have attracted particular attention thanks to their ability to contain photosensitive agents, such as silver, in relatively large concentration. The interaction of such glasses with a high repetition rate fs NIR laser leads to the formation of locally distributed fluorescent silver clusters. ${ }^{[8,9]}$ These clusters, composed of less than 20 atoms, result from multiphoton ionization associated with pulse after pulse cumulative thermal effects, allowing the reduction of silver ions and the diffusion of silver species. These clusters can act as nucleation centers for larger entities such as NPs after subsequent aggregation.

Herein, we describe how fs NIR excitation in tailored phosphate glasses can activate silver atom and ion clustering, giving rise to silver clusters. We then show how a post-irradiation thermal development allows the promotion of silver reduction and the growth of metallic NPs from the clusters acting as nucleation centers. Absorption and luminescence spectroscopies are appropriately exploited to investigate the evolution of the silver species after irradiation and thermal treatment. By analogy with photography, we emphasize on the mechanism of 
silver clustering that is initiated by oxido-reduction processes under NIR fs excitation in the amorphous network. Silver atom and ion assemblies ( $m<20$ atoms) typically correspond to the latent image, which is developed by thermal treatment to form silver NPs. Thus, we demonstrate how a thermal post-treatment completes the $\mathrm{Ag}^{+}$diffusion and silver cluster reduction, leading to the growth of metallic NPs. The results demonstrate an innovative strategy to address the problem of controlling nanoassembly processes of metal disconnected NP patterns in fully inorganic and stable hard materials. ${ }^{[10]}$ The proposed approach makes possible the fabrication of 3D metal structures in optical-quality inorganic materials.

\section{Results and Discussion}

\subsection{DLW Process: Irradiance and Pulse Number Dependence}

DLW was performed on a linearly translated glass sample with controlled speeds from 10 up to $100 \mu \mathrm{m} \mathrm{s}^{-1}$, corresponding at each position from $0.17 \times 10^{6}$ up to $1.7 \times 10^{6}$ deposited pulses (corresponding to A, B, C and D columns in Figure 1a). DLW was processed for different fluencies from 4.3 to $5.5 \mathrm{~J} \mathrm{~cm}^{-2}$ (from $I=11.25$ to $13.75 \mathrm{TW} \mathrm{cm} \mathrm{cm}^{-2}$, labeled lines 1,2 and 3 in Figure 1a). Linear structures, with lengths of $100 \mu \mathrm{m}$ and interline distances of $5 \mu \mathrm{m}$ were written, resulting in square patterns, as shown in Figure 1a. The linear polarization of the writing beam was parallel to the sample translations (and thus to the lines) during the DLW process. No polarization dependence on the written structures was evidenced. Figure 1a shows the wide-field fluorescence imaging from the laser-induced silver clusters.

Micro-transmission measurements were performed both in a pristine area of the glass and in structured zones, and gave a direct experimental access to the local differential microextinction due to the laser-induced structures. Such microextinction signals were measured on each of the 12 processed squares. They are represented in Figure 1b, while their analysis is explained in Figure $1 \mathrm{c}$ and detailed in Figure 1d. The calculations of the optical transition energies for some charged Ag clusters (up to $\mathrm{m}=14$ atoms) were performed by Ershov et al. ${ }^{[11]}$ Some of them exhibit several intense absorption bands (a)

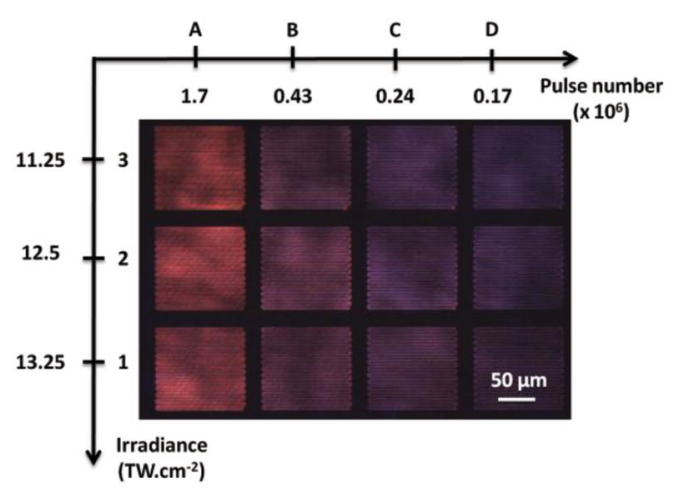

(c)

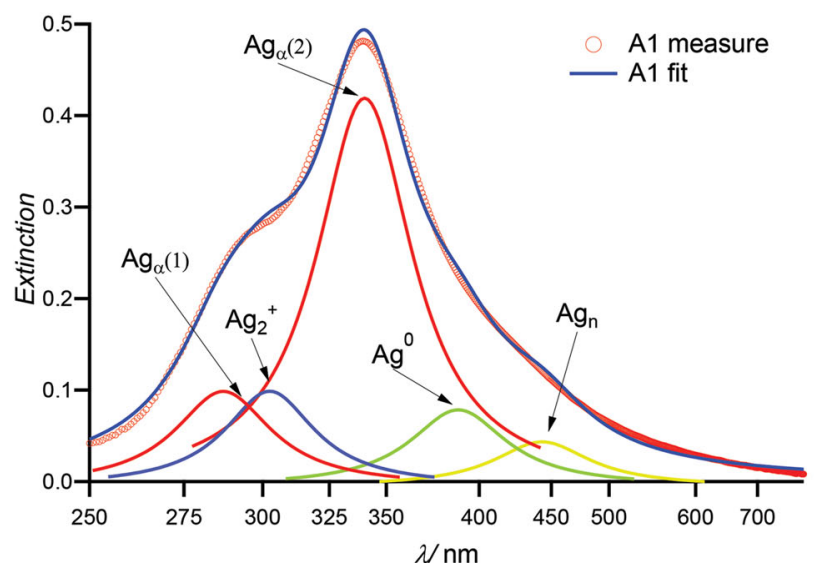

(b)

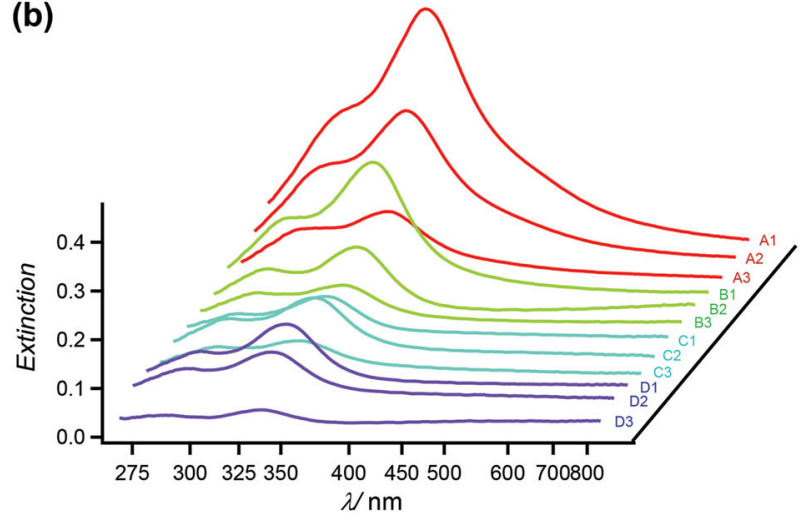

(d)

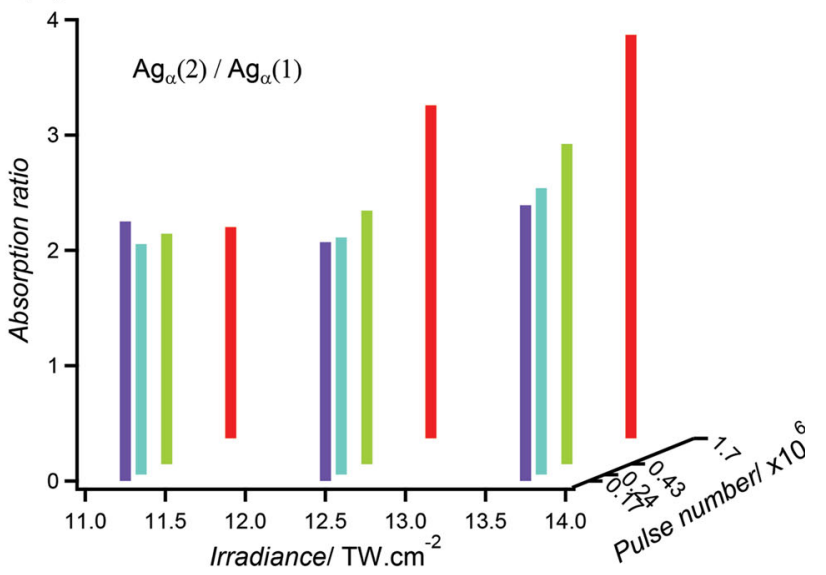

Figure 1. (a) Wide-field fluorescence imaging: fluorescence image for an excitation at $\lambda_{\text {exc }}=405 \mathrm{~nm}$ and an emission in the visible wavelength range from the laser-written zones. Irradiances are depicted along the vertical axis, and pulse numbers along the horizontal axis, for the parametric spectroscopic study detailed below. Shadow effect is due to non-homogeneous excitation of the sample. (b) Stack of the extinction spectra from the 12 laser irradiation conditions. (c) Extinction spectrum of the laser-induced Al square pattern with maximum irradiance $\left(13.25 \mathrm{TW} \mathrm{cm}^{-2}\right)$ and pulse number $\left(1.7 \times 10^{6}\right)$, and the optimal deconvolution of the spectrum into Lorentzian functions (red: larger silver clusters labeled $\mathrm{Ag}_{\alpha}$, blue: $\mathrm{Ag}_{2}{ }^{+}, \mathrm{green} \mathrm{Ag}^{0}$, and yellow $\mathrm{Ag}_{\mathrm{n}}$ ). (d) Associated $\mathrm{Ag}_{\alpha}(2) / \mathrm{Ag}_{\alpha}(1)$ absorption ratios showing a remarkable constant value and thus highly suggesting their attribution to the same silver species $\mathrm{Ag}_{\alpha}$ for any irradiation condition, except for the $\mathrm{A} 1$ and $\mathrm{A} 2$ conditions where an additional behavior appears (red color bars). 
Table 1. Amplitude, position, and FWHM values of the fi ve Lorentzian functions used for the deconvolution of the A1 pattern extinction. The $\chi^{2}$ result is equal to 0.016703 , stressing the relative good achievement of the deconvolution function.

\begin{tabular}{lccccc}
\hline $\mathrm{Ag}$ specie & Amplitude & $\begin{array}{c}\text { Position } \\
{[\mathrm{nm}]}\end{array}$ & $\begin{array}{c}\text { Position } \\
{[\mathrm{eV}]}\end{array}$ & $\begin{array}{c}\text { FWHM } \\
{[\mathrm{nm}]}\end{array}$ & $\begin{array}{c}\text { FWHM } \\
{[\mathrm{eV}]}\end{array}$ \\
\hline $\mathrm{Ag}_{\alpha}(1)$ & $0.103 \pm 0.001$ & $287 \pm 2$ & $4.32 \pm 0.06$ & $35 \pm 4$ & $0.5 \pm 0.02$ \\
$\mathrm{Ag}_{2}{ }^{+}$ & $0.104 \pm 0.001$ & $302 \pm 3$ & $4.1 \pm 0.08$ & $34 \pm 4$ & $0.45 \pm 0.02$ \\
$\mathrm{Ag}_{\alpha}(2)$ & $0.42 \pm 0.005$ & $340 \pm 2$ & $3.64 \pm 0.04$ & $56 \pm 6$ & $0.57 \pm 0.015$ \\
$\mathrm{Ag}^{0}$ & $0.084 \pm 0.001$ & $387 \pm 4$ & $3.2 \pm 0.06$ & $57 \pm 5$ & $0.47 \pm 0.014$ \\
$\mathrm{Ag}_{n}$ & $0.049 \pm 0.003$ & $442 \pm 1$ & $2.8 \pm 0.01$ & $73 \pm 7$ & $0.45 \pm 0.012$ \\
\hline
\end{tabular}

between $250 \mathrm{~nm}$ and $400 \mathrm{~nm}$. For example for the cubic $\mathrm{Ag}_{8}{ }^{2+}$ clusters, the experiment shows two transitions near $290 \mathrm{~nm}$ (s-s levels) and $325 \mathrm{~nm}$ (s-p* levels). This cluster appears to be exceedingly stable because of an even number of atoms and a moderated and even number of charges. The geometric configuration corresponds to a structural fragment of the metallic $c f c$ lattice. ${ }^{[11]}$ In our experiments, two bands located at $\lambda=287 \mathrm{~nm}(\mathrm{E}=4.32 \mathrm{eV})$ and $\lambda=340 \mathrm{~nm}(\mathrm{E}=3.64 \mathrm{eV})$ are listed as the two major extinctions named $\operatorname{Ag}_{\alpha}(1)$ and $\operatorname{Ag}_{\alpha}(2)$, respectively. As reported by Ershov et al., those bands could correspond either to $\mathrm{Ag}_{4}{ }^{2+}$ or $\mathrm{Ag}_{8}{ }^{2+}$ clusters. In our experiments, this band assignment is not only given to one cluster or another, therefore we will group the two bands signal together in one clustercalled $\operatorname{Ag}_{\alpha}$. The presence of such center $\mathrm{Ag}_{\alpha}$ was assumed for the extinction spectrum deconvolution. Defect centers such as $\operatorname{Ag}^{0}(\lambda=387 \mathrm{~nm} ; \mathrm{E}=3.2 \mathrm{eV})$ have been reported in irradiated silver-containing phosphate glasses and are widely documented. ${ }^{[12,13]}$ From Figure 1a, two other bands are also necessary for coherent modeling: one band at $\lambda=$ $302 \mathrm{~nm}(\mathrm{E}=4.1 \mathrm{eV})$ attributed to $\mathrm{Ag}_{2}{ }^{+[12,14,15]}$ and another one at $\lambda=442 \mathrm{~nm}(\mathrm{E}=2.8 \mathrm{eV})$ from the SPR of metallic silver in the studied glass. ${ }^{[16]}$ In Figure 1c, the fitted curves with these four species listed in Table 1 are in good agreement with the experimental results from the twelve irradiation conditions. From all spectra in Figure 1b (representing the different experimental irradiation conditions), these five identified transitions (with fixed spectral band positions adjusted with the A1 condition, as shown in Figure 1c) were sufficient and relevant for a very good fit, even though we are aware that a spectrum deconvolution with many functions may not only have one signification.

Amplitude ratios for the extinction peaks at $\lambda=340 \mathrm{~nm}$ $\left(\operatorname{Ag}_{\alpha}(2) ; \mathrm{s}-\mathrm{p}^{*}\right.$ transition) and $\lambda=287 \mathrm{~nm}\left(\mathrm{Ag}_{\alpha}(1)\right.$; s-s transition) are represented in Figure $1 \mathrm{~d}$. At low dose, these absorption ratios are constant at $2.2 \pm 0.1(\mathrm{~B}, \mathrm{C}$ and $\mathrm{D}$ pulse number conditions). Contrarily, for high doses (A1, A2 and A3 irradiance conditions), a net deviation of the ratio from $2.2 \pm 0.1$ is observed, around $3.5 \pm 0.1$ (bar plots in red; Figure 1d). This strongly suggests the presence of DLW-induced $\mathrm{Ag}_{\alpha}$ clusters for the low-dose depositions, while another kind of species appears for the high-dose depositions. For those writing parameters, the concentration of the laser-induced clusters drastically increases. At high DLW doses, the temperature and the number of photoelectrons increase in a non-linear way. Thanks to the cumulative effect associated with the high repetition rate of the laser, the Ostwald ripening process is most likely occurring. ${ }^{[17]}$ One also has to keep in mind that the concentration of the photoinduced clusters also depends on the number of reducing agents, such as non-bridging oxygens (NBOs) and defects. ${ }^{[18]}$ The ratio deviation is then explained by the enhancement of the absorption band around $\lambda=340 \mathrm{~nm}$, being attributed to the appearance of a new center, probably a larger cluster than $\mathrm{Ag}_{\alpha}$, which we will call $\mathrm{Ag}_{\beta}$. In addition, for the highest dose (A1 pattern; Figure 1c), another absorption band is perceptible at lower energy around $\lambda=442 \mathrm{~nm}(\mathrm{E}=2.8 \mathrm{eV})$, corresponding to the SPR of silver colloids embedded in glass. ${ }^{[16]}$

Strong DLW parameters lead to the production of silver colloids in zinc-phosphate photosensitive glasses. By analogy with photography, we have demonstrated that the laser irradiance/pulse number parameters during DLW play a key role (i) towards the processing of concentrated $\mathrm{Ag}_{\alpha}$ clusters $\left(\mathrm{Ag}_{4}{ }^{2+}\right.$ or $\mathrm{Ag}_{8}{ }^{2+}$ ) below a given threshold dose, and (ii) towards a modification and reduction of clusters into NPs above such a threshold dose.

\subsection{Thermal Development Subsequent to DLW: Extinction and Fluorescence Emission Spectroscopies}

Following DLW process, the glass was heated to $400{ }^{\circ} \mathrm{C}, 20^{\circ} \mathrm{C}$ above the glass transition temperature $\left(\mathrm{T}_{\mathrm{g}}=380{ }^{\circ} \mathrm{C}\right)$, for $2 \mathrm{~h}$. The corresponding absorption and fluorescence measurements of all laser-induced structures are depicted in Figure 2.

After thermal post-treatment, a strong SPR extinction band around $\lambda=442 \mathrm{~nm}$ is again observed for most of the laserinduced patterns. After DLW followed by thermal development around the glass transition temperature, $\mathrm{Ag}_{\alpha}$ (1) and $\mathrm{Ag}_{\alpha}$ (2) levels and $\mathrm{Ag}_{\mathrm{n}} \mathrm{SPR}$ are listed as the three major absorption features (Figure 2a,b).

Under all the DLW conditions used for the experiments, the SPR absorption increases up to an optical density of 0.71 (Figure $2 \mathrm{~b}$ for the A1 square pattern). We observe in Figure 2c that the $\operatorname{Ag}_{\alpha}(2) / \operatorname{Ag}_{\alpha}(1)$ ratios are distributed around an average value of $2.2 \pm 0.1$ and are slightly dependent on the DLW dose/ irradiance. This average value is the same as the calculated ratio for the $\mathrm{Ag}_{\alpha}$ specie after DLW only (without thermal treatment). For the writing parameters where a deviation was previously observed (red bars in Figure 1d, related to A1 and A2 conditions), the thermal development procedure restores the constant ratio $\mathrm{Ag}_{\alpha}(2) / \mathrm{Ag}_{\alpha}(1)$ around $2.2 \pm 0.1$, stressing the disappearance of the $\mathrm{Ag}_{\beta}$ species identified as larger clusters (Figure $2 \mathrm{~d}$ ). Additionally, the absorption amplitudes of such $\mathrm{Ag}_{\alpha}$ species are 
(a)

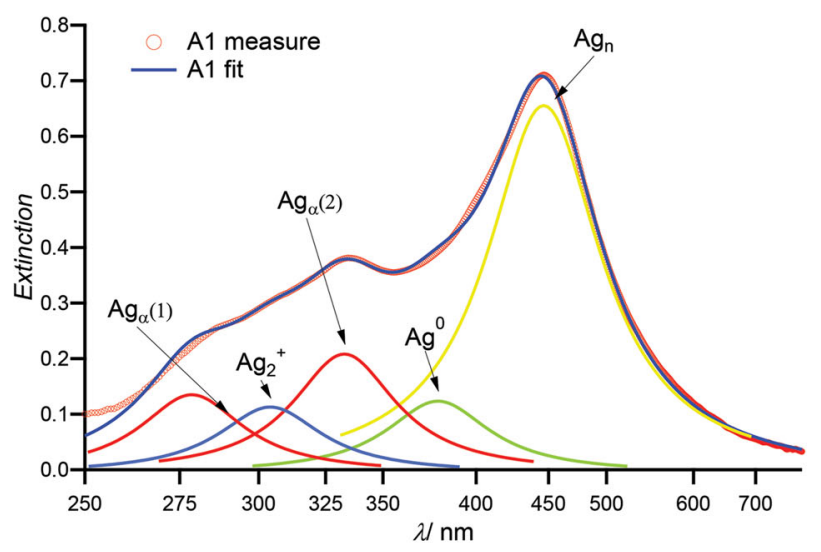

(c)
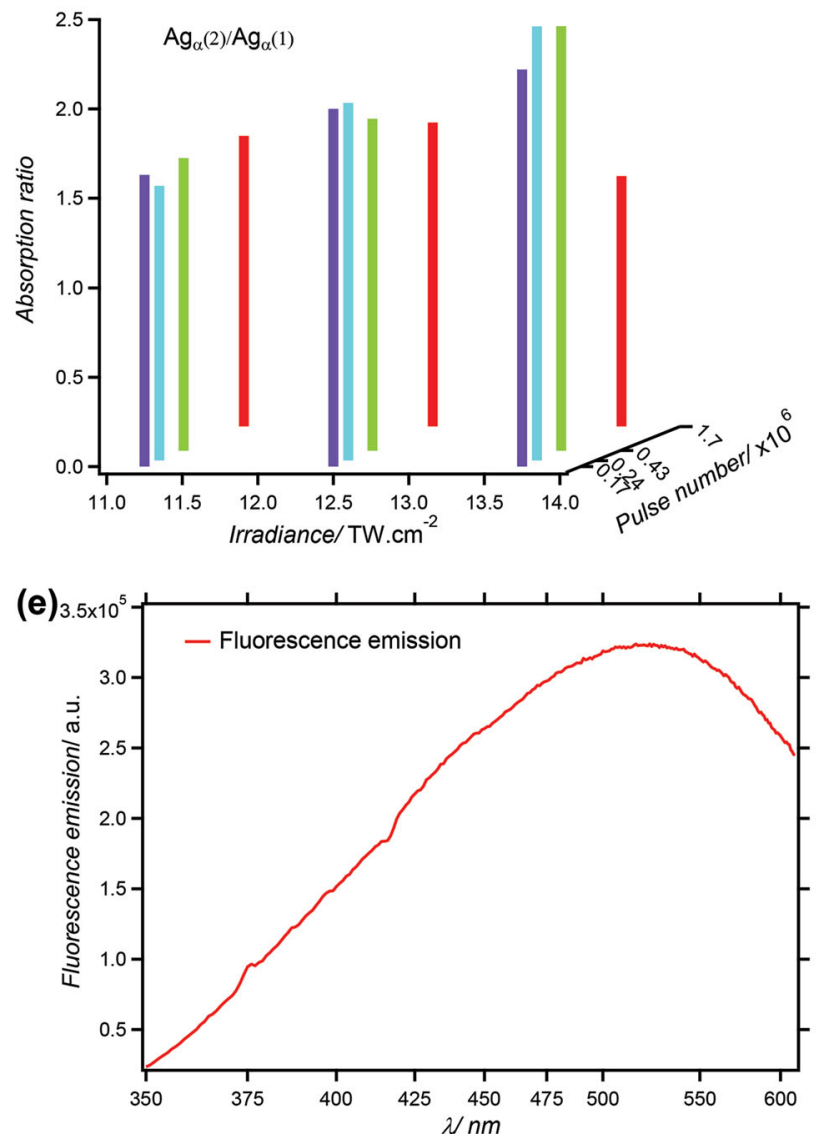

(b)

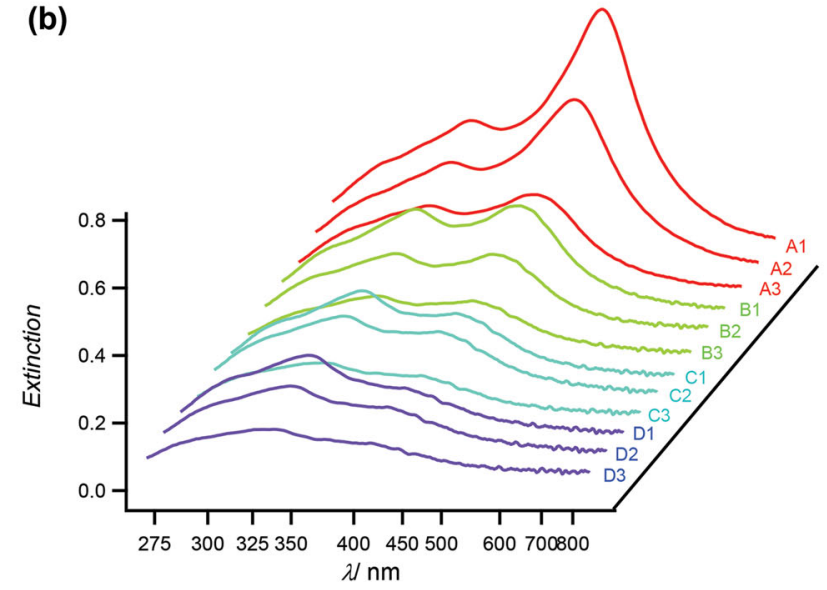

(d)

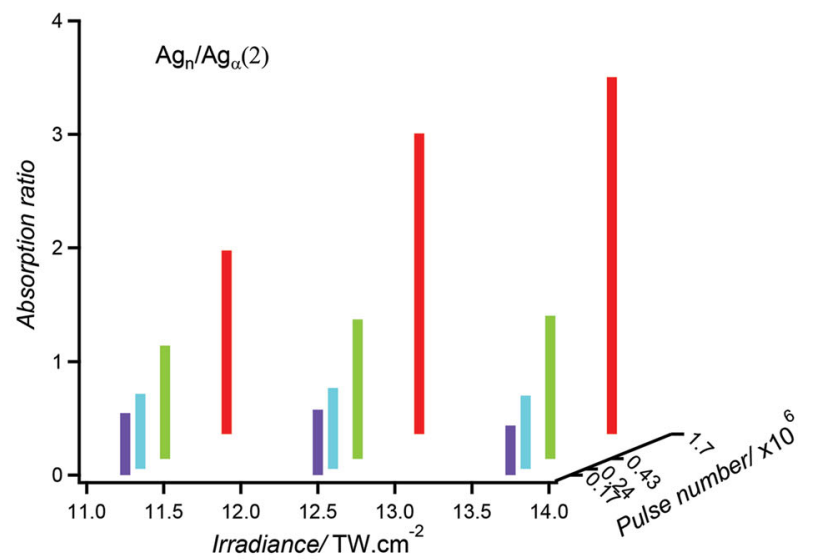

(f)

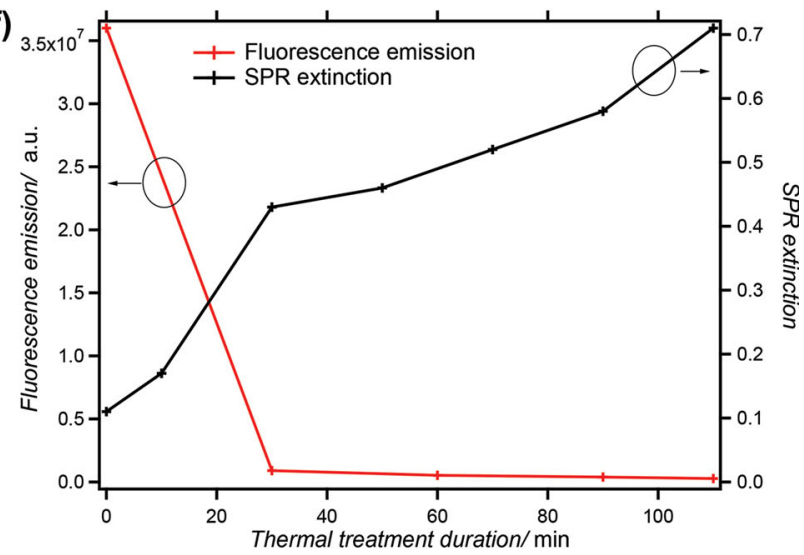

Figure 2. (a) Extinction spectra after DLW and subsequent thermal development (A1 condition - red empty circles), and fitting curve with the same five Lorentzian curves (in deep blue) as in Figure 1c. (b) Stack representation of the extinction spectra depending on the twelve explored DLW parameters. For each written pattern, amplitude ratios of the Lorentzian fitting functions: (c) $\mathrm{Ag}_{\alpha}$ (2)/Ag $/ \mathrm{g}$ (1), and (d) $\mathrm{Ag}_{\mathrm{n}} / \mathrm{Ag}_{\alpha}$ (2). (e) Corrected fluorescence emission spectrum for the highest DLW irradiance/dose parameters (A1 pattern), under $\lambda_{\text {exc }}=325 \mathrm{~nm}$. (f) Evolution of the related $A g_{\alpha}$ fluorescence emission intensity (from the integration of the whole emission spectrum) and correlated SPR absorption band amplitude at $\lambda=442 \mathrm{~nm}$, as a function of the thermal development duration.

divided by 2 after thermal treatment $(0.2$ instead of 0.4$)$. Comparatively, we observe that $\mathrm{Ag}_{\mathrm{n}} / \mathrm{Ag}_{\alpha}(2)$ ratios vary from 0.5 up to 3 and increase with the deposited irradiances/pulse numbers. We clearly evidence here that these $\mathrm{Ag}_{\beta}$ act as precursors for NP formation. Such precipitation of silver clusters into NPs occurs after thermal development only for some DLW sensitization parameters. As the mobility of $\mathrm{Ag}^{+}$ions and silver clusters in glasses is assumed to be low, compared to the results obtained in solution, ${ }^{[11]}$ Ostwald ripening is most likely occurring. Prior to thermal treatment, the DLW dose is the trigger 


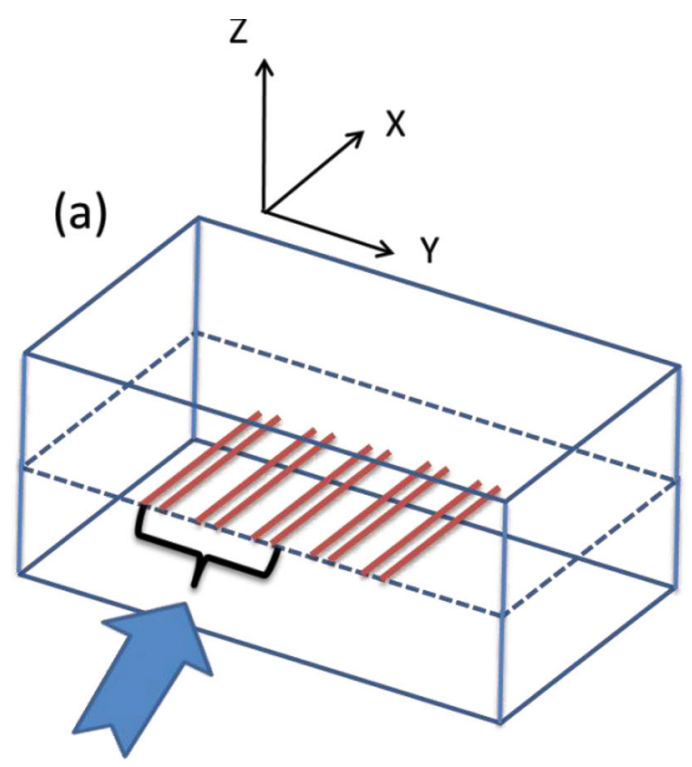

HRSEM image

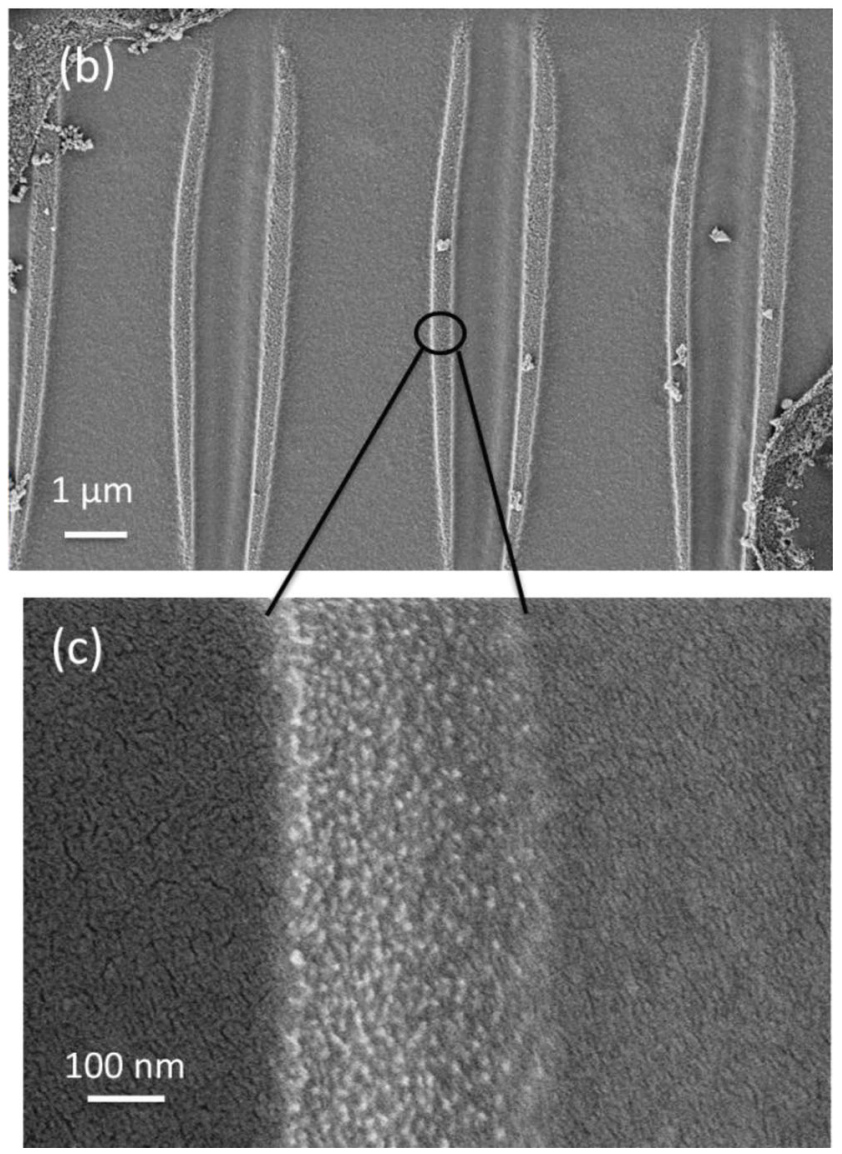

Figure 3. (a) Sketch representing laser-written lines in a selected plane in the volume of the glass; (b) HRSEM (secondary electron mode) image of the cross-section of Ag NP structures induced after DLW and thermal treatment; (c) 100 000x magnification HRSEM (back-scattering mode) image showing the local high concentration of silver inside the frame of Figure $3 b$. factor to allow the formation of intermediate state clusters $\mathrm{Ag}_{\beta}$ that can lead to the precipitation of metallic NPs.

The different deconvolutions of the absorption responses reveal a slight shift of the $\mathrm{Ag}_{\mathrm{n}}$ absorption band. The modification of the SPR is explained by the variation in the NP concentration embedded inside the glass according to the DLW pre-sensitization, corresponding to changes of the so-called filling factor. ${ }^{[19]}$ We assume the existence of some positively charged $\mathrm{Ag}_{\alpha}$ in the near vicinity of the silver NPs visible by energy shift and also partial damping of the SPR absorption. ${ }^{[20,21]}$ Nonetheless, the damping of the SPR is compensated by the strong increase of the silver NP concentration, leading to the observed drastic increase of the SPR absorption.

The fluorescence and absorption evolutions versus the thermal development duration of such laser-induced patterns for several sequences are represented in Figure 2f. We observe the very intense $\left(3.10^{5}\right.$ counts $\left.\mathrm{s}^{-1}\right)$ fluorescence emission prior to annealing (Figure 2e). A broadband emission is observed with a maximum emission around $\lambda=530 \mathrm{~nm}$. In phosphate glasses, $\mathrm{Ag}_{\alpha}$ have shown a broadband fluorescence emission around $\lambda=500 \mathrm{~nm} \cdot{ }^{[22]}$ After the first $30 \mathrm{~min}$ thermal development session, a significant diminution of the global intensity is observed (800 counts $\mathrm{s}^{-1}-$ not represented in Figure 2). The fluorescence emission intensity attributed to the $\mathrm{Ag}_{\alpha}$ centers strongly decreases after NP formation (Figure 2f). Correlatively, we observe a decrease (by half) of the extinction bands related to $\mathrm{Ag}_{\alpha}$ clusters in the UV-blue spectral window, indicating that approximately half of these clusters has disappeared, which is not sufficient to account for the global decrease of the fluorescence. The strong decrease of the luminescence intensity is mostly attributed to the quenching of the luminescence due to the vicinity of the NPs, which will be further detailed below.

Figure 3a presents the linear patterns produced by moving the sample in the $x$ direction at constant speed, resulting in the generation of a double-line pattern. The laser interaction process in this glass can be viewed as a paintbrush and an eraser working together at the nanoscale. The paintbrush acts on the edges of the laser beam and the eraser works in the center. ${ }^{[8]}$ This is explained as a writing process with two distinct thresholds. A writing threshold creates the clusters along the moving direction and a photodissociation threshold erases the clusters at the maximum intensity of the laser beam. In this way, arbitrary 3D patterns can be written inside the photosensitive glass. The sample was then cut perpendicular to the lines ( $\gamma z$ plane) and the surface was etched in diluted $\mathrm{HNO}_{3}$ acid, in order to reveal the nanostructures at the surface. We then performed a thermal treatment in order to get the development of silver NPs. Figure $3 \mathrm{~b}$ presents the high-resolution scanning electron microscope (HRSEM) image of the cross-section of the photoinduced structures, where one can distinguish both walls of the structure. Figure $3 \mathrm{c}$ shows a backscattered electron-mode image. This acquisition mode is more sensitive to the atomic weight of the analyzed elements. Silver NPs are observed to be spatially confined in 120 nm-thick walls in the $x y$ writing plane (Figure 3c), while we previously estimated the wall length (in depth) to be around $8 \mu \mathrm{m}$ (Figure $3 \mathrm{~b}$ ). We therefore consider the NPs to be confined in a $21.6 \mu \mathrm{m}^{3}$ elementary cell volume. In Figure $3 c$, the areas exhibiting high silver concentration 
(a) Redox potential

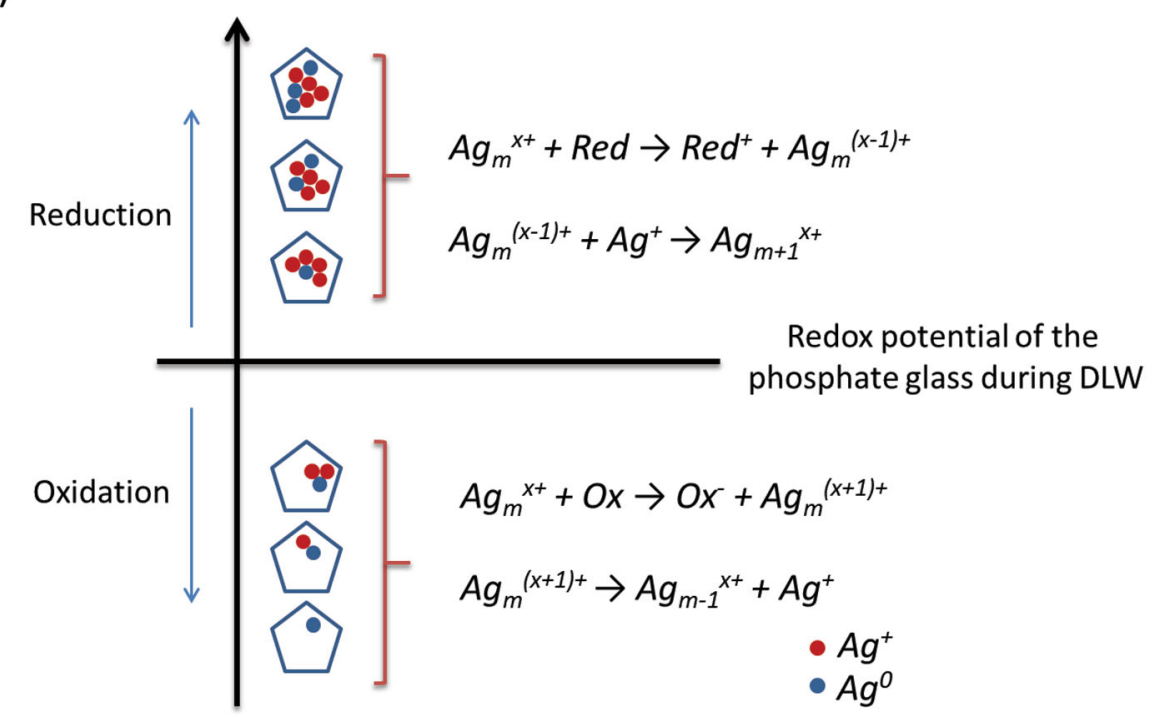

(b)

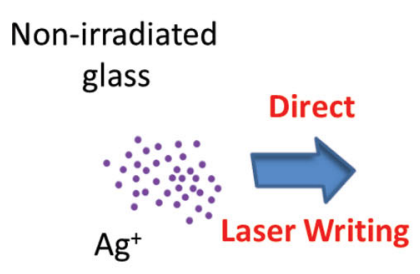

Laser-glass interaction volume

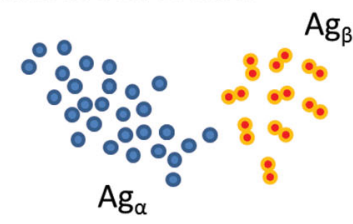

NPS

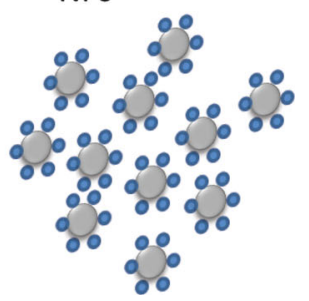

Scheme 1. (a) Representation of the mechanism of silver clustering from oxido-reduction processes initiated by fs NIR irradiation, showing the stabilization and growth of sufficiently large clusters (with redox potentials higher than the surrounding phosphate matrix redox potential) or, opposingy, the dissolution and disappearance of small clusters (with lower redox potentials). (b) Schematics representing the two-step process leading to the formation of silver NPs after DLW followed by thermal development inside silver containing zinc phosphate glasses.

correspond to nodules with a 5 to $15 \mathrm{~nm}$ diameter size, homogeneously spread in the volume of the wall. This size most likely corresponds to the NP size, thus allowing us to assume that these nodules are the silver NPs. We have conducted FDTD calculations based on the measured SPR extinction spectra, while considering the dielectric medium (the refractive index of the glass is $n=1.62$ in the visible range) and silver-related constants, which gave us an estimation of the NP size. We obtained an average NP size of $5 \pm 1 \mathrm{~nm}$. The NPs are localized and confined at the same location as the laser-induced clusters. These results strengthen the idea that some of these clusters (mostly the $\mathrm{Ag}_{\alpha}$ clusters) behave as nucleation centers.

After $2 \mathrm{~h}$ of thermal development, an SPR-related optical density of 0.71 is measured, corresponding to an extremely high effective linear absorption coefficient of $1750 \mathrm{~cm}^{-1}$. The NP concentration must be considerably high to obtain such absorption values. Considering the absorption cross-section of $5 \mathrm{~nm}$ spherical silver NPs to be approximately $10 \mathrm{~nm}^{2},{ }^{[23]}$ we calculated the density of NPs embedded in the considered elementary cell. After high dose DLW procedure, we obtained nearly isolated NPs (about 20 in $21.6 \mathrm{\mu m}^{3}$ ). After DLW followed by thermal treatment, we estimated the number of NPs to be around 1000 in the same volume. Nonetheless, taking into account a potential interaction of the NPs with the $\mathrm{Ag}_{\alpha}$ clusters, the SPR absorption may result in a strong decrease, a shift of the maximum to longer wavelengths as well as broadening of the band, ${ }^{[20]}$ so that this NP density estimation could be largely underestimated, increasing therefore the actual NP concentration (this concept is not included in the scope of this article). As a matter of fact, following this approximation, we consider the interparticle distance to be at the nanometric scale between two neighboring silver particles. Many groups have led research in energy transfer between NPs and molecular systems. ${ }^{[24]}$ Their work refers in particular to strong fluorescence quenching when the molecule/system of interest is placed nearby metallic objects $(<5 \mathrm{~nm})$ and high fluorescence enhancement when the distance is over $10 \mathrm{~nm} \cdot{ }^{[25]}$ After several post-DLW thermal developments, for a total duration of $2 \mathrm{~h}$, we observe the strong decay of the global intensity in a homothetic manner and finally the extinction of the fluorescence emission. As presented in Figure $2 \mathrm{f}$, the probable quenching of the fluorescence emission increases with the $\mathrm{Ag}_{\mathrm{n}}$ density (filling factor). In the same Figure $2 \mathrm{f}$, we note that after $30 \mathrm{~min}$ of thermal development, the NP concentration gives rise to a dramatic reduction of the fluorescence emission. Such diminution can be attributed (i) to the conversion of silver clusters into NPs, and (ii) to Förster 
energy transfer between the silver NPs and all the other surrounding remaining silver species.

\subsection{Discussion}

The results presented in this article bring information in order to reach 3D patterning of silver NPs in tailored zinc-phosphate glasses: (i) the laser processing dose can be adjusted by controlling the irradiance and duration of the irradiation to develop the space-selective production of nucleation centers and then (ii) the space-selective growth and subsequent reduction of the $\mathrm{Ag}_{\beta}$ species into silver NPs can be activated and performed by thermal development.

In studies conducted on mainly silicate glasses, a thermal treatment above the glass transition temperature leads to bulk homogeneous precipitation of NPs within the glass volume. This originates from the silver cluster concentration and the number of non-bridging oxygen atoms (NBOs), which play the role of reducing agents. ${ }^{[17]}$ On the contrary, in the presented phosphate glass, thermal treatment without DLW does not induce silver particle formation. This is due to a sufficiently low redox potential of the phosphate matrix, in relation to the absence of reducing agents, as shown in Scheme 1a.

To describe the evolution of the different silver species after laser irradiation and thermal treatment, we propose a twostep mechanism. First, hole centers (mostly $\mathrm{Ag}^{0}$ ) act as mobile species to form $\mathrm{Ag}_{\alpha}$ clusters with surrounding $\mathrm{Ag}^{+}$ions, as presented in Scheme 1b. By analogy with film-based photography mechanism, when the laser dose (i.e. the number of pulses) is high enough, the concentration of low-nuclearity species is sufficient to allow their clustering in a low quantity of $\mathrm{Ag}_{\alpha}$ species. An intermediate state of $\mathrm{Ag}_{\beta}$ clusters is necessary to allow the formation of silver NPs. Second, diffusion processes are at the origin of the growth and reduction of $\mathrm{Ag}_{\beta}$ species in $\mathrm{Ag}_{n}$ NPs. Over tens of atoms, silver aggregates tend to adopt a metallic behavior, evidenced by the SPR, which dominates the extinction spectrum. For a temperature of some hundreds of degrees Celsius, most of the free electrons and holes recombine slowly. Espiau et al. ${ }^{[14]}$ suggested that the redox potential of silver clusters increases with their nuclearity. They indicate the existence of a critical aggregate size of 3 silver entities, below which, clusters are unstable with respect to reoxidation (hence re-dissolution) due to the holes being released by glass defects. Above this size threshold, silver clusters appear stable, and metallic NPs may grow by $\mathrm{Ag}^{+}$diffusion in water from the cluster and in situ reduction. Nonetheless, more investigations are necessary to identify the relation between the initial number of defect centers (related to both the quantity of electron-hole pairs generated during the irradiation and the temperature increase) and the final amount of stabilized $\mathrm{Ag}_{\alpha}$ clusters.

Femtosecond lasers generate two luminescent parallel lines when focused and then translated in the photosensitive glass sample. ${ }^{[2,27]}$ More complex inscriptions can be achieved when synchronously displacing the sample in the three directions in space. For example, we coded a helicoïdal path or movement with respect to the laser beam. Figure 4 presents the reconstructed images of 2D stacks of confocal fluorescence microscopy

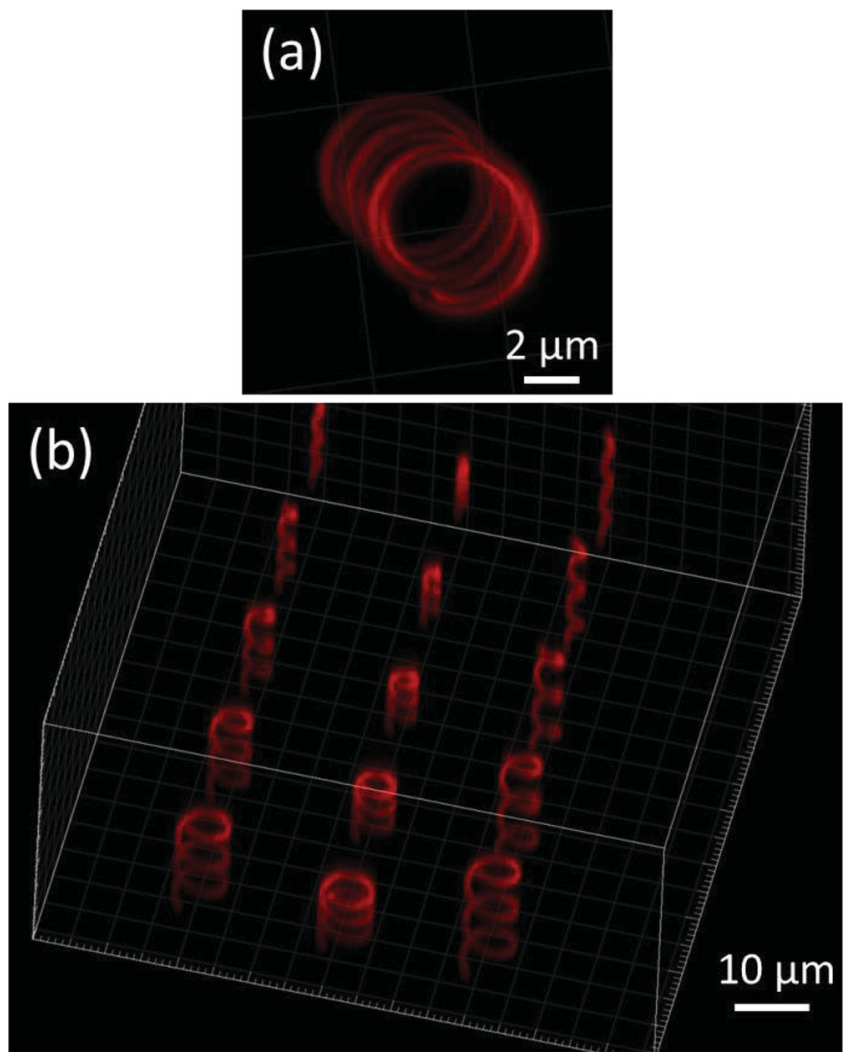

Figure 4. Reconstructed confocal fluorescence microscopy image (100x objective, $N A=1.4, \lambda_{\text {exc }}=405 \mathrm{~nm}, \Delta \lambda_{\text {em }}=420-700 \mathrm{~nm}$ ) from 3D laserinduced helicoidally-distributed emitting $\mathrm{Ag}_{\alpha}$ silver clusters. (a) Single and (b) array of helicoïdal patterns with different dimensions (transverse radius and axial period).

images. As previously demonstrated, the NPs developed by thermal treatment subsequent to DLW are located at the same location as the silver clusters. We have therefore confirmed here the ability to generate complex 3D shapes of NP structures embedded in dielectric transparent glass materials, opening up the design and the fabrication of new photonic devices.

\section{Conclusion and Perspectives}

In this article, we have reported a very detailed study of the correlative microscopy for both absorption and fluorescence emission that resulted from NP formation in silver-containing phosphate glasses after fs DLW processing and thermal posttreatment. First, we observed the formation of silver clusters after fs laser irradiation. Second, we highlighted the formation of NPs by the diffusion of small silver elements in the sample. The analogy with photography has been evoked; sufficient fs laser irradiation doses are the trigger parameter to obtain a latent image of large enough silver clusters to be stable, while the developed image after thermal treatment leads to plasmonic silver NPs in the dielectric medium.

Absorption spectroscopy has evidenced the species resulting from DLW sensitization of phosphate glassy sample. Unique $\mathrm{Ag}_{\alpha}$ centers are proposed, that could correspond to $\mathrm{Ag}_{4}{ }^{2+}$ or $\mathrm{Ag}_{8}{ }^{2+}$ clusters, to serve as nucleation centers for 
NPs precipitation. These resulting $\operatorname{Ag}_{\alpha}$ species offer the chemical stability to further be reduced into metallic silver with tens of atoms, thanks to silver ion and atom mobility. Importantly, giving multi-arametric absorption spectroscopy information, we have observed that a minimal DLW dose is necessary to develop plasmonic NPs with the thermal post-irradiation treatment.

Fluorescence spectroscopy has confirmed the existence of $\operatorname{Ag}_{\alpha}$ clusters and also revealed the extinction of fluorescence after long thermal post-irradiation treatment $(2 \mathrm{~h})$. It is probable that the strong diminution of the fluorescence emission correlated to the growth of $\mathrm{Ag}_{\alpha}$ clusters into NPs, but also to emission quenching effects of these $\mathrm{Ag}_{\alpha}$ clusters because of the vicinity of the NPs.

The results presented herein have attractive perspectives for the development of future applications via the direct 3D patterning of metal disconnected nanoparticle structures in glass below the diffraction limit $(\sim 100 \mathrm{~nm})$. In-depth patterning is achievable with transparent materials, while electronic lithography only proposes surface structuring of non-transparent silicon substrates. In comparison with other methods, ${ }^{[28]}$ our concept is not limited by the different technological phases and allows in a simple two-step process, to achieve real 3D disconnected subwavelength metallic features. The direct process enables the synthesis of homogeneously dispersed and encapsulated nanoparticles in glass, preventing oxidation and pollution from the atmosphere. Such materials exhibiting strong plasmon resonances could be used as substrates for surface enhanced Raman scattering (SERS) or fluorescence enhancement for high-sensitivity sensing devices. The design of $3 \mathrm{D}$ chiral structures would also be of prime importance for photonics applications. Beyond that, the processing of $3 \mathrm{D}$ nanometer-scale metallic structures in glass will give access to meta-properties. The lateral resolution $(\sim 100 \mathrm{~nm})$ and the lattice periodicity are compatible with requirements for the design of metamaterials in the NIR range.

\section{Experimental Section}

Preparation: Tailored zinc phosphate glass (with $4 \%$ of molar $\mathrm{Ag}_{2} \mathrm{O}$ ) was fabricated using a conventional elaboration method as described in ref. [8] After quenching, the glass was annealed, cut with a typical thickness of $1 \mathrm{~mm}$ and polished to optical quality. DLW was conducted using a microscopy setup equipped with a NIR fs laser and a high-precision 3D translation stage (a KGW:Yb oscillator, $2 \mathrm{~W}, 10 \mathrm{MHz}, 470 \mathrm{fs}$ at $1030 \mathrm{~nm}$, T-Pulse 200, Amplitude Systèmes; a Mitutoyo objective APO PLAN NIR, 100× NA 0.7; a high-precision 3D translation XMS-50 stages, MicroContrôle, $50 \mathrm{~nm}$ resolution). DLW was performed inside the bulk at typically $170 \mu \mathrm{m}$ below the surface by linearly moving the glass sample at controlled speeds. Thermal annealing was carried out by heating the sample at $400^{\circ} \mathrm{C}$ in air, slightly above the glass transition temperature $T_{g}=380^{\circ} \mathrm{C}$ for $2 \mathrm{~h}$.

Characterization Techniques: Absorption measurements were performed in bright-field using a 'CRAIC Technologies' microscope equipped with a Xenon lamp and a condenser as a white light source. A conjugated 10x magnification microscope objective was used to collect the transmitted light through the laser-induced structures. Fluorescence spectrum measurements were performed with a high spectral resolution spectrometer (LabRAM HR800 from Jobin-Yvon) between 350 and $600 \mathrm{~nm}$. A 150 grooves. $\mathrm{cm}^{-1}$ grating was used for the fluorescence spectral dispersion in the visible region after excitation with a UV He-Cd laser source ( $30 \mathrm{~mW}, \lambda=325 \mathrm{~nm}, \mathrm{TEM}_{00}$, Kimmon). A UV enhanced 36× NA 0.5
Cassegrain objective was used for UV excitation and visible epi-collected fluorescence. Localized 3D fluorescence imaging was performed with a confocal microscope $\left(\lambda_{\text {exc }}=405 \mathrm{~nm}\right.$, SP8, Leica). A High Resolution Scanning Electron Microscope (HRSEM Gemini Ultra 55 - Field emission gun - resolution $1.7 \mathrm{~nm}$ at $1 \mathrm{kV}$ ) was used for irradiated sample imaging. Two different detectors were used: in lens back-scattered electrons and secondary electrons modes to produce images of the sample.

\section{Acknowledgements}

This work was carried out with financial support from the French State, managed by the French National Research Agency (ANR) in the frame of the "Investments for the future" Programme IdEx Bordeaux (ANR-10IDEX-03-02), Cluster of excellence LAPHIA. We are grateful to Christophe Boyaval from IEMN in Lille for HRSEM measurements. Confocal images of helicoïdal structures were performed at the Photonic Imaging platform of the Bordeaux Imaging Center (UMS 3420 CNRS). The authors are grateful for the help in FDTD calculations from Lumerical Solutions, Inc.

[1] A. Royon, Y. Petit, G. Papon, M. Richardson, L. Canioni, Opt. Mat. Exp. 2011, 1, 866.

[2] R. R. Gattass, E. Mazur, Nat. Photonics 2008, 2, 219.

[3] J. Qiu, M. Shirai, T. Nakaya, J. Si, X. Jiang, C. Zhu, K. Hirao, Appl. Phys. Lett. 2002, 81, 3040.

[4] Q. Zhao, J. Qiu, X. Jiang, C. Zhao, C. Zhu, Opt. Exp. 2004, 12, 4035.

[5] A. Stalmashonak, G. Seifert, H. Graener, J. Opt. A: Pure Appl. Opt. 2009, 11, 1464.

[6] G. Seifert, M. Kaempfe, K. J. Berg, H. Graener, Appl. Phys. B 2000, $71,795$.

[7] P. Zijlstra, J. W. M. Chon, M. Gu, Nature 2009, 459, 410.

[8] M. Bellec, A. Royon, B. Bousquet, K. Bourhis, M. Treguer, T. Cardinal, M. Richardson, L. Canioni, Opt. Exp. 2009, 17, 10304.

[9] M. Bellec, A. Royon, K. Bourhis, J. Choi, B. Bousquet, M. Treguer, T. Cardinal, J.-J. Videau, M. Richardson, L. Canioni, J. Phys. Chem. C 2010, 114, 15584

[10] K. K. Vora, E. Mazur, J. Vis. Exp. 2012, 27.

[11] B. G. Ershov, G. V. Ionova, A. A. Kiseleva, Rus. J. Phys. Chem. 1995, 69, 239.

[12] W. Zheng, T. Kurobori, J. Luminescence 2011, 131, 36.

[13] S. Fan, C. Yu, D. He, K. Li, L. Hu, Rad. Meas. 2011, 46, 46.

[14] R. Espiau de Lamaestre, H. Béa, H. Bernas, J. Belloni, J. L. Marignier, Phys. Rev. B 2007, 76, 205431.

[15] W. Zheng, T. Kurobori, Y. Miyamoto, H. Nanto, T. Yamamoto, Rad. Meas. 2011, 46, 1402.

[16] U. Kreibig, M. Vollmer, Optical Properties of Metal Clusters, Springer, Berlin, Germany 1995.

[17] D. Tatchev, A. Hoell, M. Eichelbaum, K. Rademann, Phys. Rev. Lett. 2011, 106, 085702.

[18] A. Simo, J. Polte, N. Pfander, U. Vainio, F. Emmerling, K. Rademann, J. Am. Chem. Soc. 2012, 134, 18824.

[19] A. Podlipensky, A. Abdolvand, G. Seifert, H. Graener, Appl. Phys. A 2005, 80, 1647.

[20] P. Mulvaney, T. Linnert, A. Henglein, J. Phys. Chem. 1991, 95, 7843.

[21] M. Kaempfe, G. Seifert, K. J. Berg, H. Hofmeister, H. Graener, Eur. Phys. J. D 2001, 16, 237.

[22] K. Bourhis, A. Royon, M. Bellec, J. Choi, A. Fargues, M. Treguer, J. J. Videau, D. Talaga, M. Richardson, T. Cardinal, L. Canioni, J. Non Cryst. Solids 2010, 356, 2658. 
[23] P. Billaud, J. R. Huntzinger, E. Cottancin, J. Lermé, M. Pellarin, L. Arnaud, M. Broyer, N. Del Fatti, F. Vallée, Eur. Phys. J. D 2007, 43, 271.

[24] Y. C. Chen, C. Y. Gao, K. L. Chen, T. H. Meen, C. J. Huang, J. Nanomat. 2013, 841436.

[25] S. Kühn, H. Ulf, L. Rogobete, V. Sandoghar, Phys. Rev. Lett. 2006, 97, 017402.
[26] L. Canioni, M. Bellec, A. Royon, B. Bousquet, T. Cardinal, Opt. Lett. 2008, 33, 360 .

[27] A. Royon, K. Bourhis, M. Bellec, G. Papon, B. Bousquet, Y. Deshayes, T. Cardinal, L. Canioni, Adv. Materials 2010, 22, 5282.

[28] C. M. Soukoulis, M. Wegener, Nat. Photonics 2011, 5, 523. 